\title{
SUITABLE DEM ASSESSMENT STUDY FOR DATA SCARCE CITIES TOWARDS URBAN EXTREME AND NUISANCE FLOOD MAPPING
}

\author{
Anjaneyulu Akkimi ${ }^{\mathrm{a}, *}$, Subashisa Dutta ${ }^{\mathrm{a}}$ \\ ${ }^{a}$ Department of Civil Engineering, Indian Institute of Technology Guwahati, Guwahati, Assam, India. \\ (a.anjaneyulu, subashisa)@iitg.ac.in
}

Commission IV, WG IV/10

KEY WORDS: Urban Flood, DEM, ALOS AW3D, ALOS PALSAR, SRTM, ASTER, NED

\begin{abstract}
:
The high-resolution accurate topography data should be used for extreme and nuisance flood inundation modeling and mapping in cities, but not available for many cities, including most developed countries. It is necessary to study and identify an alternate open-source topographic model that satisfies high-resolution topography datasets' conditions. We analyzed the open-source DEMs visually, elevation histogram statistics, streams and watershed identification, contour statistics, Topographic Wetness Index, and vertical accuracy of other medium-resolution DEMs compared with high-resolution LiDAR data over New York City to determine alternative open-source Digital Elevation Model in the context of urban flood modeling. In high urban sprawl areas, in the context of flood mapping, our findings have shown that the medium resolution DEMs predicted similar to high-resolution DEMs with the same linear errors around RMSE 25-35ft and LE90 30-40ft. Overall, the ALOS AW3D performed better than other open-source DEMs. Even though SRTM predicted well, it inducted smoothness in DEM where more buildings were located. It noted that ALOS PALSAR DEM is not suitable for any urban studies. ASTER DEM has also shown good agreement with LiDAR and observed elevations, but it induced by noise while processing. Finally, it can be suggested that the ALOS AW3D can be used as an alternative source for urban flood modeling which represented footprints of buildings even though it performed average in vertical accuracy.
\end{abstract}

\section{INTRODUCTION}

To map the flood inundations, for extreme and nuisance floods in urban areas, the topography has become an essential data. The evolution of DEM which represents the topography of the earth changed existed condition of hydrological modeling. Many global and quasi-global DEMs are available all over the world. ASTER GDEM (Tachikawa et al., 2011), SRTM (Farr et al., 2007), ALOS AW3D (Tadono et al., 2016) are freely available global datasets, TanDEM-X (Krieger et al., 2007) is both commercial $(10 \mathrm{~m}, 30 \mathrm{~m})$ and freely available (30m) global data. Globally accepted that high-resolution topographic models are necessary to map accurate flood inundations and forecasting (Sanders, 2007). To achieve this many highend technologies (Optical Photogrammetry, radar-based interferometry and airborne LiDAR etc) developed and adopted towards the direction of producing high-resolution DEM datasets such as IKNOS, SPOT, CARTOSAT (5m-actual acquisition resolution by ISRO), TanDEM-X, ALOS AW3D (5m-actual acquisition resolution by JAXA), LiDAR (sub-centimetre level) cloud points etc. At the same time, many researchers disagree that high-resolution datasets must for hydrological modeling, and the source is very important rather than its spatial resolution ( $\mathrm{Li}$ and Wong, 2010). In other words, besides solving the problems they create other complexities such as huge computation time during flood prediction, it misinterprets the wrong streams where flow path obstructs by man-made artefacts (Qin et al., 2018). To get exact stream lines the datasets has to be preprocessed which is complex and time expensive. Although many researchers are carried out to assess the accuracy of DEMs towards fluvial floods, river modeling etc., (Zhang et al., 2019, Fereshtehpour and Karamouz, 2018, Li and Wong, 2010, Sanders, 2007) but very few studies are attempted for urban flood mapping and modeling (Kelleher and McPhillips, 2020). It is very important to study and identify the suitable freely available medium resolution DEM for urban flood modeling and mapping in data scare urban areas. Also, researchers consider smooth topography for flood modeling, but in urban landscape the man-made artifacts such as buildings and roads needed in flood modeling. These footprints are important to simulate the flood water movement and velocities in urban landscape. So, the accurate DEM which represents both highresolution features and flood obstacles should be identified. In the present study, it is attempted to identify an alternate suitable DEM which approximately matches with the high-resolution dataset such as the LiDAR-based topography model. To achieve the goal a few steps are developed to select the suitable alternate medium resolution DEM flood mapping in urban areas. In this work, the freely available DEMs such as ALOS AW3D 30m (JAXA acquired at $5 \mathrm{~m}$ and then re-sampled to $30 \mathrm{~m}$ ), ALOS PALSAR 12.5, ASTER 30m, and SRTM 30m were compared with high resolution datasets LiDAR 1ft, SLR 3m, CUDEM $1 / 9$ arcsec and observed buildings elevations by using proposed steps to identify the suitable alternative DEM which satisfies the characteristics of high resolutions DEM data.

\section{STUDY AREA, DATASETS AND DATA PRE-PROCESSING}

\subsection{Study Site}

In this study, New York City and a part of it (New York county), is considered (Figure 1) as the study area because of the availability of all commercial and free datasets with high to medium

\footnotetext{
* Corresponding author
} 


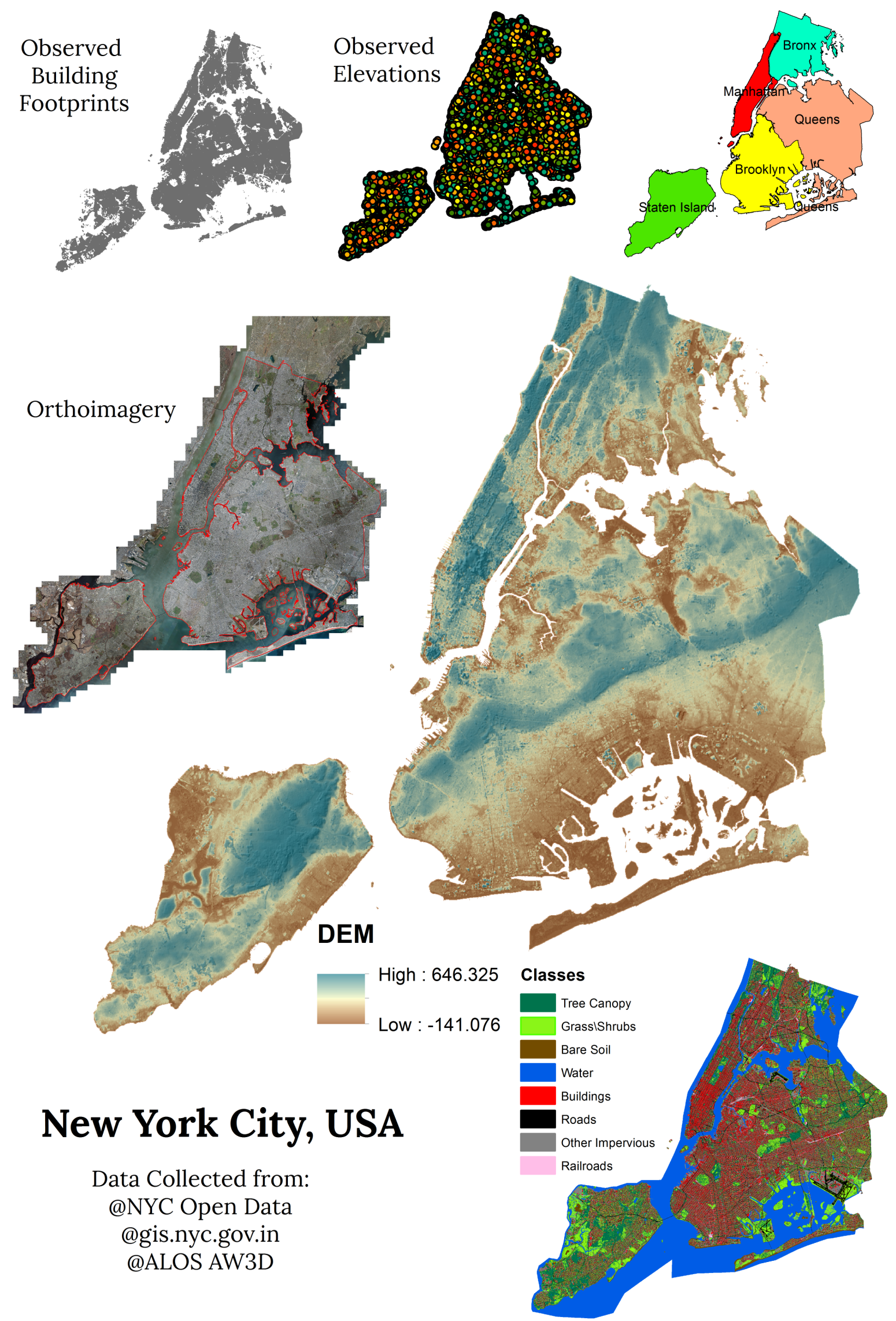

Figure 1. Five county of New York City; with observed building foot prints, elevations of ground and man-made structures, Land Use Land Cover map and ALOS AW3D shaded relief map (illumination from $315^{\circ} \mathrm{N}$ and $20^{\circ}$ above horizon) 
resolution topographic models such as LiDAR, NED, NOAA DEMs, SRTM etc. This city has the observed building roof heights, spot and other artefacts elevation datasets. This would be useful to identify the alternative sources of freely available medium resolution DEMs which portraits approximate topography similar to the surveyed (high resolution) one. New York is one of the most urbanized, populated and near to coastal belt, it is prone to pluvial and low-lying (Nuisance) floods. More details of the study area can be found in other literature (Kelleher and McPhillips, 2020).

\subsection{Datasets and its Description}

High resolution $1 \mathrm{ft}$ LiDAR bare-earth data was obtained from NYC open data portal provided by the Department of Environmental Protection (DEP) and the Department of Information Technology and Telecommunications (DoITT). The other datasets considered for the study are listed in Table 1 . The observed building roof heights, ground elevations and other artefacts elevation (Planimetric data) of 2019 and 2018 were obtained from the NYC Open data portal. This dataset contains each building roof height and other artefacts with ground elevation in the study region.

\subsection{Data Pre-Processing}

All datasets were projected into one coordinate system (NAD 1983 State Plane) in ArcMap 10.6.1 to perform raster algebra and compare datasets. The orthometric height conversion was not performed on topographic models because all datasets referenced the geoid system. The undulations in lakes and other water bodies are also crucial in urban areas, so water masking was not performed on any collected DEM. The sink filled DEMs were considered only for watershed delineation; for other objectives, sink filling was not performed. In this study, point observed elevations are considered to calculate the vertical accuracy of all topographic models, so the data points are not resampled.

\subsection{Software}

The GRASS-GIS version 7.8 (GRASS Development Team, 2019) and ArcMap 10.6.1 were used to process, view, delineate watersheds, identify streams, and perform raster calculations. Matlab 2019a was used to analyze the datasets statistically.

\section{METHODOLOGY}

The following steps describe the methodology to explore alternate free or medium resolution DEM, which closely matches high-resolution data in data scares cities.

\subsection{Statistical Matrices of Elevations and Slopes}

Descriptive statistics of elevations such as mean, median, standard deviation, skewness, kurtosis, and other statistics were calculated and presented as histograms and tables for all topographic models. The slope maps were generated, and descriptive statistics of slopes are discussed in histogram curve with table (Grohmann, 2018, Pipaud et al., 2015).

\subsection{Watershed Delineation and Stream Identification}

The stream network in urban watersheds shows floodwater flow in gullies, streets, and natural streams passing through the cities. The total number of streamlines and their total length, number of sub-watersheds, and cumulative area can indicate the extent of artifacts and noise included in the topographic models. The visual inspection of stream network at roads, streets, line breaking near culverts, bridges, and any obstructions indicates the accuracy of identifying flow paths. In this study, the watersheds were delineated, and streams were extracted in the study area with ArcHydro (Maidment and Morehouse, 2002) extension tool in ArcMap 10.6.1. The sinks were filled, and then flow direction was identified. The flow accumulation was calculated based on an optimized threshold in terms of the number of cells and area. The threshold value of approximately $0.62 \mathrm{~km}^{2}$ $\left(6.67 \mathrm{Mft}^{2}\right)$ was used to determine the flow accumulation. The procedure suggested by (Li and Wong, 2010) used to identify thresholds. Finally, the DEMs were compared with the extracted stream lengths, number streams, and visual inspection.

\subsection{Contour lines analysis}

The analysis of contour lines length and the number of contour lines (Grohmann, 2018) was used to identify the ruggedness in the topography introduced by the DEM development technique. The artificial artifacts can introduce the ruggedness in the topographic models as a noise. The contour lines analysis also gives the whole idea about representing different DEMs over the same area. The contour lines were generated using ArcMap 10.6.1 then the total number of contours and cumulative length of contours were calculated for each selected DEM. Then identified how different DEMs are performing over the same area and which free source DEM matches with highresolution DEM.

\subsection{Topographic Wetness Index (TWI) based comparison}

The locations of nuisance floods in cities are linked to topography, which can be predicted with TWI and Sink depths. The locations where high TWI value and large sink depths are having more chances to experience the nuisance floods (Kelleher and McPhillips, 2020). In this study, the TWI based comparison was performed to identify the alternative medium resolution DEM. The procedure for estimating TWI was adopted from literature (Kelleher and McPhillips, 2020) at each cell according to below-given equation (1)

$$
T W I=\ln \left(\frac{a}{\tan \beta}\right)
$$

where $\quad a=$ upslope area

$$
\tan \beta=\text { local slope }
$$

TWI value can not be calculated where slopes are null. So, those cells were replaced with a minimal value (similar to 0.001 )

\subsection{Elevations Discrepancies}

To analyze elevation differences, the vertical difference of each DEM was quantified relative to planimetric observed data during 2019 and 2018 observed by DoITT. The elevation difference analysis can be used to access the vertical accuracy of DEM in 


\begin{tabular}{|c|c|c|c|c|}
\hline \multirow{3}{*}{$\begin{array}{l}\text { Dataset } \\
\text { LiDAR }\end{array}$} & Spatial Resolution & Type & Agency & Source \\
\hline & $1 \mathrm{ft}$ & DTM & $\begin{array}{l}\text { DEP and } \\
\text { DoITT }\end{array}$ & NYC Open data portal \\
\hline & $1 \mathrm{~m}$ & & & \\
\hline NED & $\begin{array}{l}1 / 3 \operatorname{arcsec}(10 \mathrm{~m}) \\
1 / 9 \operatorname{arcsec}(30 \mathrm{~m})\end{array}$ & - & USGS & 3DEP (https://viewer.nationalmap.gov/basic/) \\
\hline CUDEM & $1 / 9 \operatorname{arcsec}(30 \mathrm{~m})$ & - & & \\
\hline SLR & $3 \mathrm{~m}$ & - & NOAA & https://coast.noaa.gov/dataviewer/ \\
\hline CONED & $3 \mathrm{~m}$ & - & & \\
\hline ALOS AW3D & $30 \mathrm{~m}$ & DSM & JAXA & $\begin{array}{l}\text { https://www.eorc.jaxa.jp/ALOS/en/aw3d30/ } \\
\text { index.htm }\end{array}$ \\
\hline ALOS PALSAR & $12.5 \mathrm{~m}$ & - & JAXA & $\begin{array}{l}\text { Alaska Satillite Facility (https://asf.alaska. } \\
\text { edu/) }\end{array}$ \\
\hline SRTM & $30 \mathrm{~m}$ & DSM & NASA & https://dwtkns.com/srtm30m/ \\
\hline ASTER GDEM & $30 \mathrm{~m}$ & DSM & NASA & $\begin{array}{l}\text { LP DAAC (https://search.earthdata.nasa. } \\
\text { gov/search) }\end{array}$ \\
\hline Building Heights & - & - & DEP and & \\
\hline Ground Elevations & - & - & DoITT & NYC Open data portal \\
\hline Planimetric Data & - & - & & \\
\hline
\end{tabular}

Table 1. Datasets considered for the study, its resolution and available source

terms of ME, RMSE, STDE and linear errors LE90 and LE95 (Zhang et al., 2019).

$$
\begin{gathered}
\text { MeanError }(M E)=\frac{1}{N} \sum_{i=1}^{N} \Delta h_{i} \\
R M S E=\sqrt{\frac{1}{N} \sum_{i=1}^{N} \Delta h_{i}^{2}} \\
S T D E=\sqrt{\frac{1}{N-1} \sum_{i=1}^{N}\left(\Delta h_{i}-\overline{\Delta h}\right)} \\
\text { Median }=Q_{\Delta h}(0.5) \\
\text { LE } 90=Q_{|\Delta h|}(0.9) \\
\text { LE95 }=Q_{|\Delta h|}(0.95)
\end{gathered}
$$

$$
\begin{array}{ll}
\text { where } & \Delta h_{i}=\text { Difference of Elevation } \\
N=\text { Total number of valid points } \\
Q_{\Delta h}(0 . x)=\text { Quantiles at } 10 x \%
\end{array}
$$

\section{RESULTS AND DISCUSSION}

The results of the six components of analysis mentioned in the previous section (section 3) are presented and discussed in the below sections.

\subsection{Analysis by Visual Inspection}

The level of urban features such as buildings, roads, trees, streams, and water bodies (lakes and ponds) resolved by selected topographic models can be seen visually in their shaded relief images. Figure 2 shows the shaded relief images of each selected DEM. At 30m spatial resolution, the building footprints are barely seen in NED and ALOS PALSAR, indicating smooth DEMs (DTM). The collected LiDAR data and CONED are bare earth models, which does not show the building footprints; instead, it created artificial sinks at the place of buildings. The ALOS AW3D 30m and ASTER show clear indications of human-made artifacts than SLR, CUDEM, NED 1m, and NED $10 \mathrm{~m}$ even though its resolution is medium. In between high-raised buildings, the ALOS AW3D does also not represent clearly, which may be due to non-penetration of signal (large $\mathrm{b} / \mathrm{H}$ ratio) and optical signal shadow effect around buildings. SRTM DEM shows smoothness or the same value in data at Manhattan region where the cluster of high raised buildings are located, which may be due to the layover and shadow effect of radar signal near surroundings of the buildings.

Finally, the LiDAR (bare earth data), NED10m, NED 30m, ALOS PALSAR, and CONED show no evidence of humanmade artifacts introduced in the production process. In contrast, SLR, CUDEM, NED 1m, and SRTM show minor artificial artifacts. ALOS AW3D 30m and ASTER shows Anthropocene artifacts clearly except in between high-raised buildings, but ASTER shows more ruggedness in the dataset.

\subsection{Descriptive Statistics of DEM Elevations and Slopes}

The descriptive statistics of all topographic models are presented as histograms with the table is shown in Figure 3. In general, all histograms of selected topography models showed similar curves with a bimodal distribution, one peak is at $-5 \mathrm{ft}$ (coastal belt), and another is around 10-40ft. All DEMs showed the first peak at the exact location, but for the second peak, they disagreed slightly. The ALOS PALSAR and SRTM are slightly shifted towards the right, with the second peak is around $20 \mathrm{ft}$. The ASTER DEM showed its peak at 40ft, which reasonably matched the observed building elevations collected by NYC Open data during 2019 by photogrammetry and human observations. The LiDAR, which is bare earth data after excluding artifacts showed a high peak at negative elevations.

Slope maps for some of the selected DEMs are shown in Figure 4. Slope maps are helpful to identify the quality of DEMs, which enhances the difference in elevations. ALOS AW3D clearly showed more steeper slopes around urban sprawls, mainly at Manhattan region, which is the downtown of New 


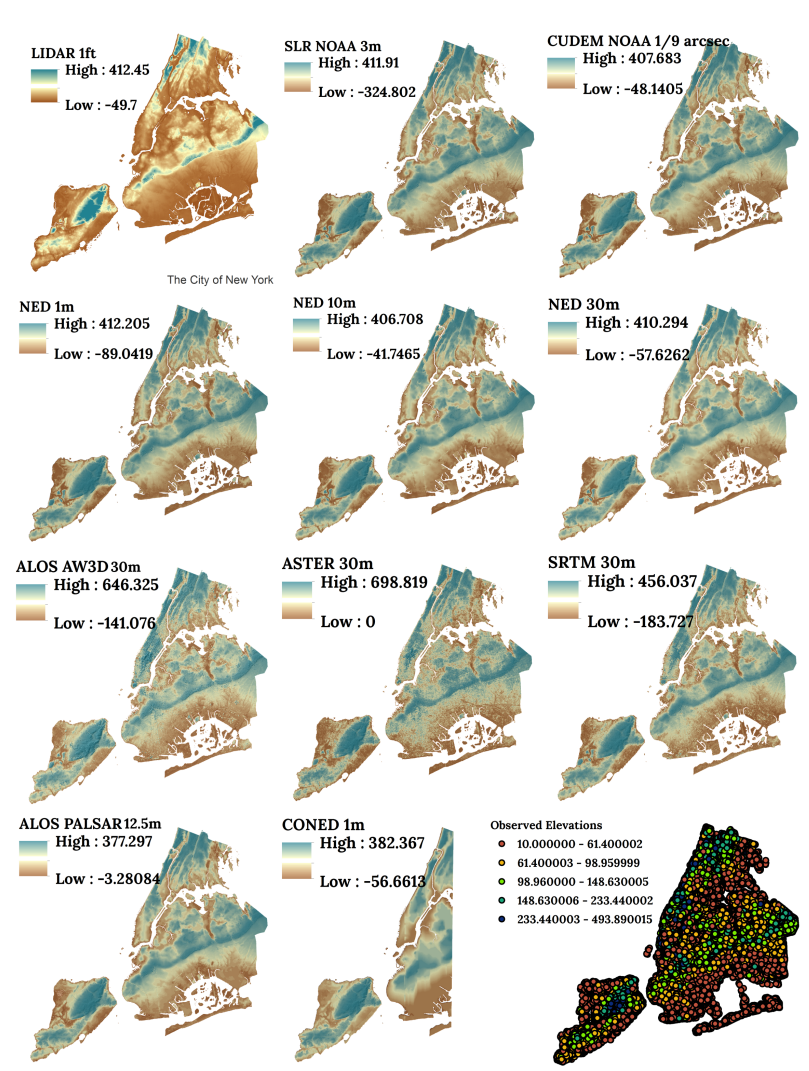

Figure 2. Shaded relief images of all DEMs at city of New York, USA (illumination from $315^{\circ} \mathrm{N}$ and $20^{\circ}$ above horizon)

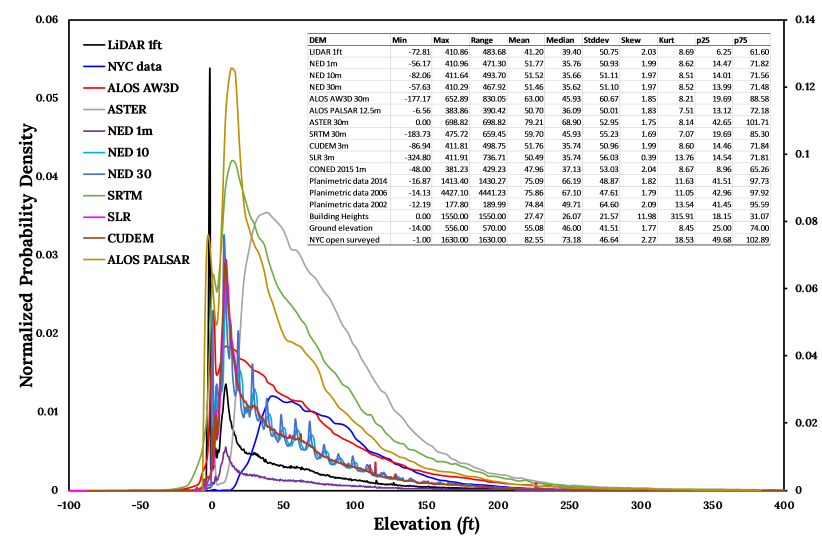

Figure 3. The distribution of elevation values for all considered datasets with histogram curves and statistical matrices table

York, than other high-resolution datasets. The ASTER and SRTM have also shown relatively little steeper slopes around the buildings. The NED 10,30m, and ALOS PALSAR did not indicate steeper slopes, indicating that these DEMs represent the smooth topography than rugged surfaces.

Figure 5 shows the histogram curves of slopes derived from selected topography models. SLR, NED 1m, and CUDEM showed steeper slopes than other datasets due to their high spatial resolution. Many studies identified that the steepness in slope decreases as DEM resolution becomes coarser (Grohmann, 2018, Grohmann, 2015, Chen and Zhou, 2013, Chow and Hodgson, 2009) and scientifically, it is true. However, in contrast, the maximum slope value in medium resol-

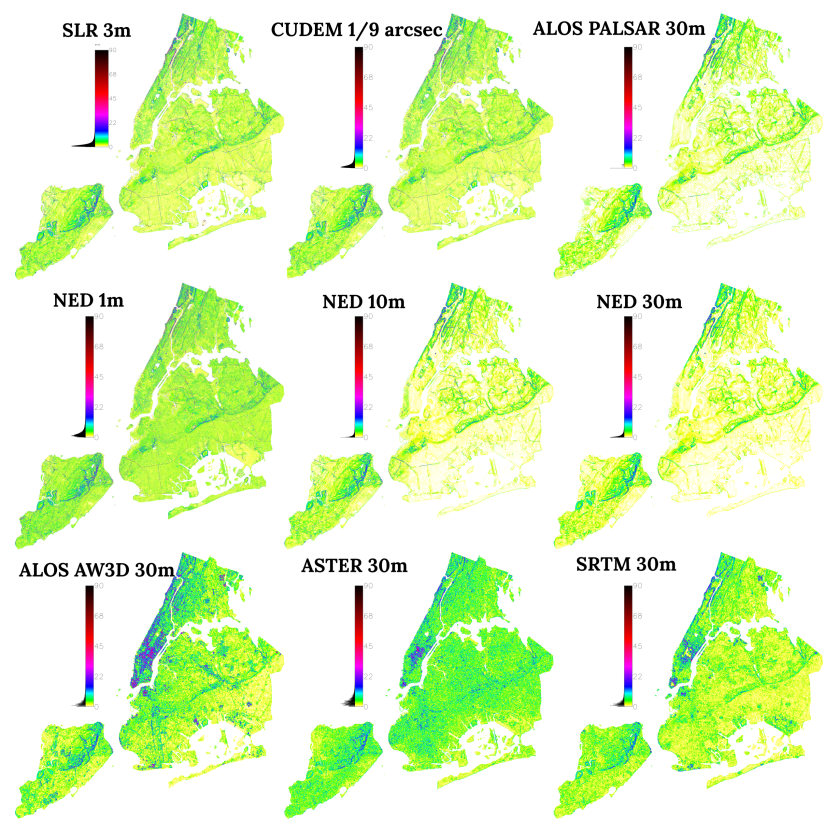

Figure 4. Slope maps for some selected DEMs over study area

ution DEMs such as ALOS AW3D, ASTER, and SRTM also showed nearer to high-resolution datasets.

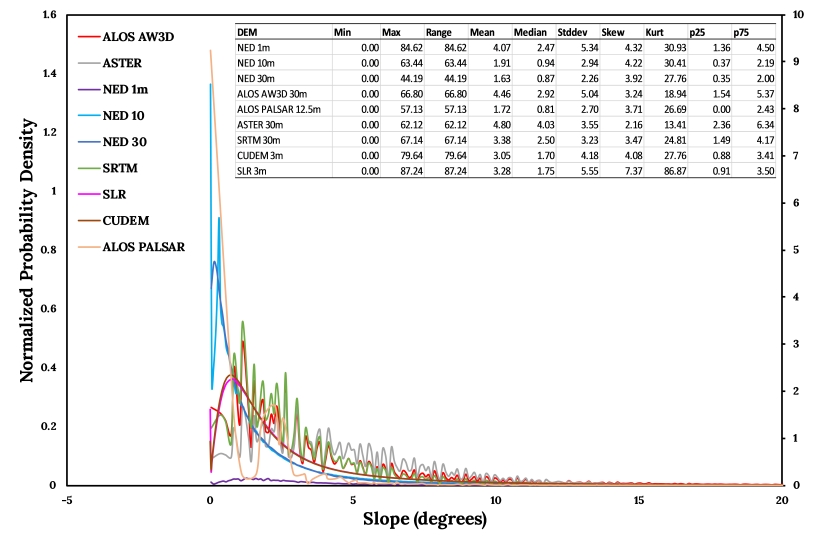

Figure 5. The distribution of slope values for all considered datasets with histogram curves and statistical matrices table

The slope histograms of ALOS AW3D, ASTER, and SRTM DEMs slightly shifted to the right and showed a more significant number of steeper slopes. Overall, all DEMs showed the same tendency towards slope identification in New York city.

\subsection{Watershed Delineation and Stream Identification}

The watersheds and streams are generated by following the steps provided in the methodology. The derived stream network and watersheds shapes are shown in Figure 6. The highresolution DEMs misregistered the stream when the stream is encountered with artifacts such as a culvert, bridge, or cluster of human-made artifacts.

In Figure 6, one of such misregistration is shown in a red circle. The high-resolution datasets such as SLR, CUDEM, NED $1 \mathrm{~m}$ showed that the extracted stream follows the train track ( $\mathrm{N}$ line) from the N-W direction to the Norward Ave road in the S-E direction instead of following the steepest slope path. The obstruction (bridges) encountered at Gowanus underpass and Brooklyn 


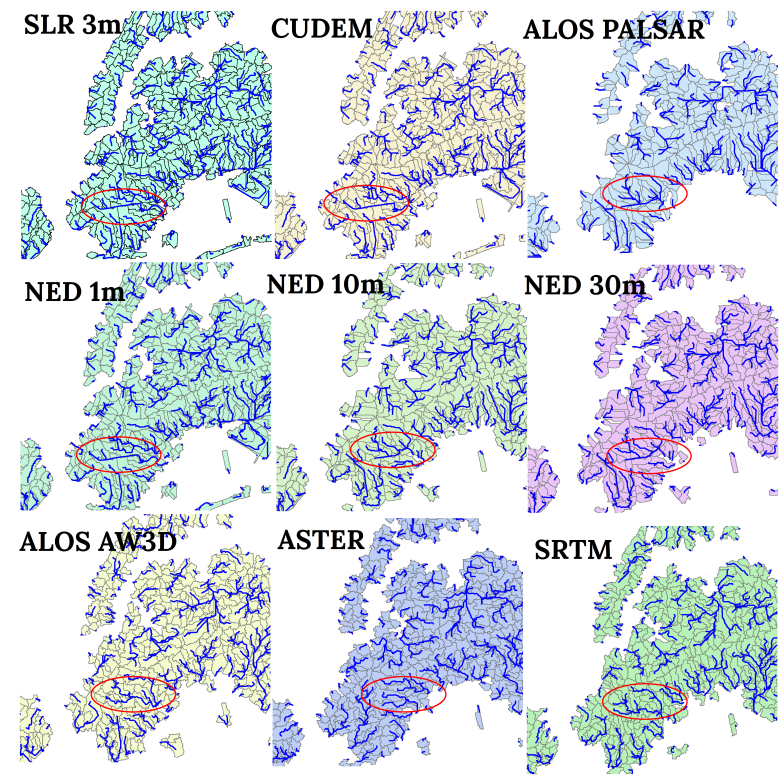

Figure 6. The stream network and watersheds extracted for selected topographical models

junction might have caused the stream diversion. While ALOS PALSAR, NED 10, and 30m DEMs represented the identical streamlines and watersheds.

However, the comparison of streams by visual inspection is difficult. The extracted streams and watersheds were evaluated by their cumulative length and number extracted for different DEMs to find alternative DEMs. Figure 7 and 8 show summary plots of the cumulative sum of stream lengths, its counts, number of watersheds, its cumulative area, and watershed perimeter length. The more stream length, watershed area, watershed length, and its high count represent whether the DEM is ruggedly introduced with artifacts or a noisy DEM, while the smooth DEMs produce lower lengths and area at the same number of counts. The high-resolution DEM also represents the high cumulative length and count due to its ability to represents more features.

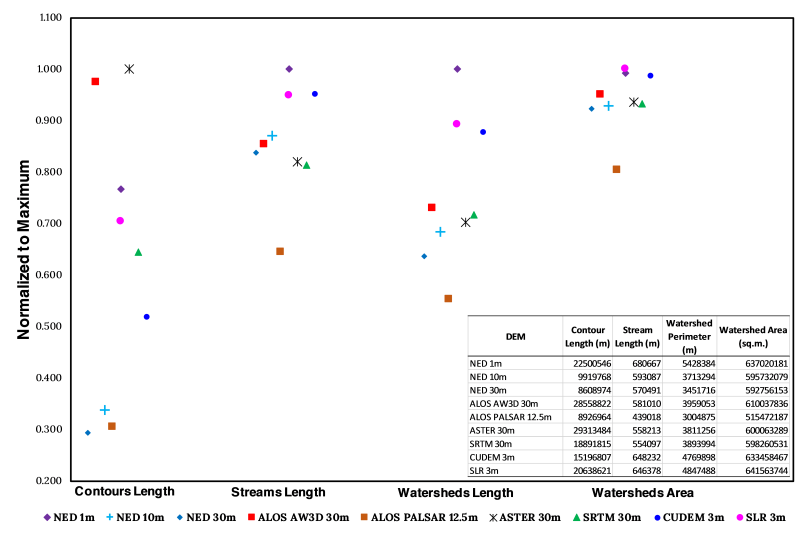

Figure 7. Cumulative sum of stream length, watershed area, watersheds perimeter length and contour length

The CUDEM, NED 1m, and SLR showed more stream length and watershed perimeter, while ALOS PALSAR $12.5 \mathrm{~m}$ showed low values even though its high spatial resolution. ALOS

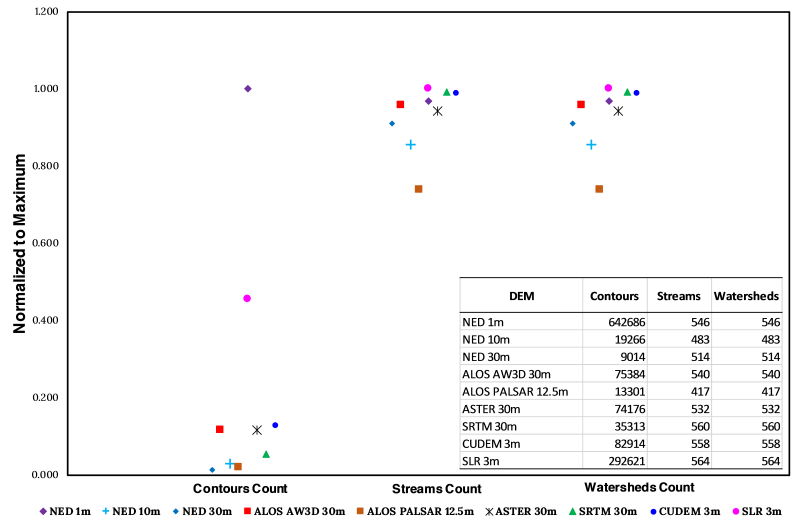

Figure 8. Cumulative sum of stream count, number of watersheds, and contour count

AW3D represented the same as NED products, while ASTER and SRTM showed similar tendencies in both streams and watersheds. In terms of total watershed area, all DEMs have given same area except ALOS PALSAR. In cumulative counts, ALOS PALSAR represented with low values and nearer to NED $10 \mathrm{~m}$ data, indicating these two DEMs are behaving similarly. Interestingly the medium resolution DEMs such as ALOS AW3D, ASTER, and SRTM produced the same number of counts in streams and watersheds, which is more than NED $1 \mathrm{~m}$ spatial resolution ( $\mathrm{Li}$ and Wong, 2010). It seems the medium resolution DEMs predicted quite similar to high resolution in terms of the number of streams, watersheds, and its lengths in the selected study area.

\subsection{Contours Analysis}

The analysis of contour lines gives the idea of how different DEMs represent the same study area differently. The contour length and counts also represent similar to streams and watersheds. The noisy DEMs produce more contour length and counts, while smooth DEMs produce low length at the same number of counts (Grohmann, 2018). The contours were produced at $10 \mathrm{ft}$ vertical elevation interval for each DEM at its original resolution. The length of contours and its counts for some selected DEMs are shown in Figures 7-8. The ALOS AW3D and ASTER produced a high number in contour length at the same number of contour count contrast to other datasets, which reveals these two datasets are introduced with humanmade artifacts or noise during its processing. While NED 10m, $30 \mathrm{~m}$, and ALOS PALSAR produced low contour lengths at the same number of contours, which reveals again these DEMS are smooth topography models excluded with artifacts such as buildings and trees. The CUDEM, SLR, NED 1m, and SRTM produced the same contour length and number.

\subsection{Topographic Wetness Index Analysis}

Figure 9 shows the topographic wetness index (TWI) generated map at Midtown Manhattan. The TWI is calculated by following the steps mentioned in 3.4.

The higher TWI indicates the higher chances of getting nuisance to extreme floods in urban areas (Kelleher and McPhillips, 2020). SLR and ALOS AW3D showed the same higher value, while NED 10m and NED 30m showed strip lines in TWI maps, indicating its smoothness and small valley streams. The ASTER and SRTM showed complete larger values, which means 


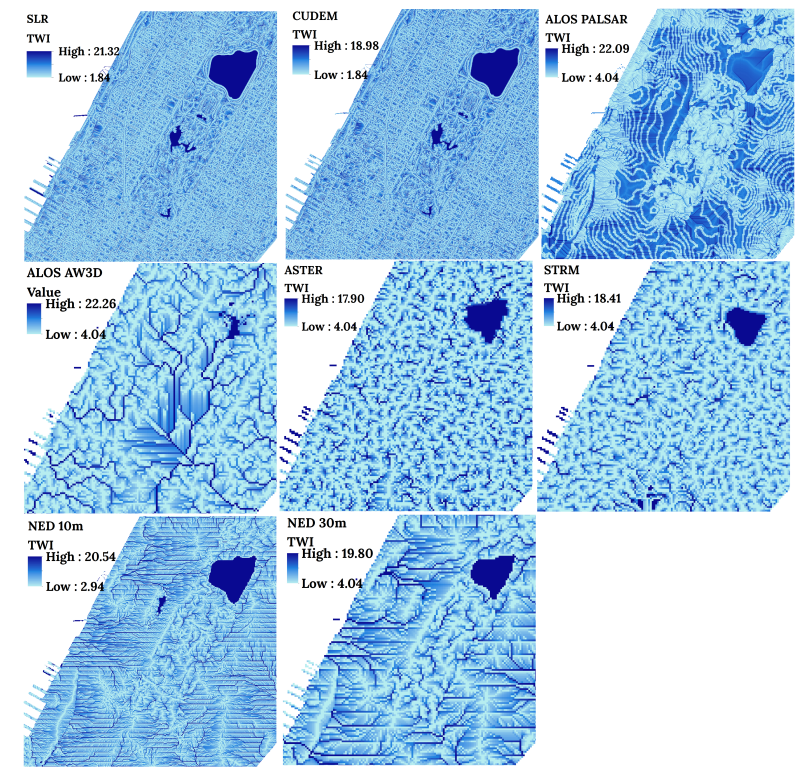

Figure 9. Generated Topographic Wetness Index map for Midtown Manhattan, New York city

the total area has more chances to get nuisance floods, but they represented TWI of water bodies in the park similar to highresolution DEMs. The ALOS AW3D has shown similar to SLR and CUDEM at midtown and south-west of park. The ALOS PALSAR misrepresented TWI over the total area even though it is high resolution. More analysis for more regions should be done to identify better DEM in the context of TWI.

\subsection{Difference in Elevations}

The elevation differences between the two topographic models are helpful to evaluate the vertical accuracy of DEMs and identify the morphological and land-use changes in any area. Here the vertical accuracy of DEM is assessed by calculating the Difference of DEMs (Elevation Discrepancies) about the observed building elevations collected during 2019 by DoITT. The histograms of elevation difference with table is shown in Figure 10.

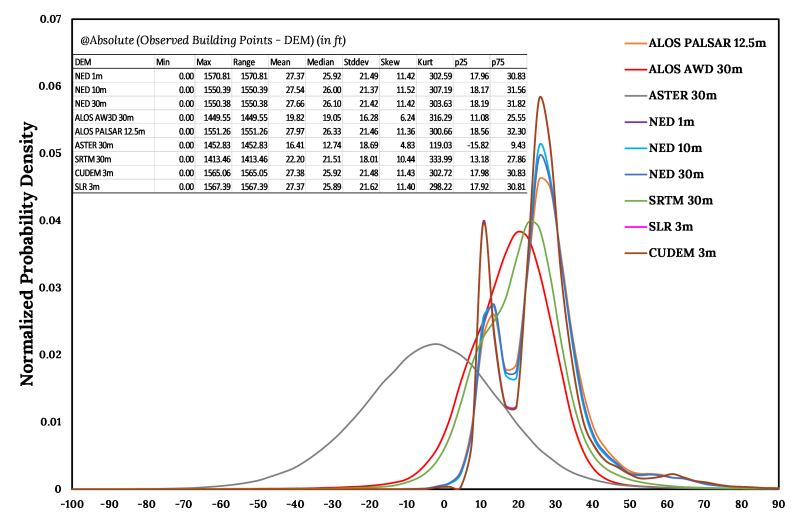

Figure 10. The distribution of elevation differences for all considered datasets with histogram curves and statistical matrices table

The medium resolution DEMs ALOS AW3D, ASTER, and SRTM showed unimodal distribution, and all other DEMs fol-

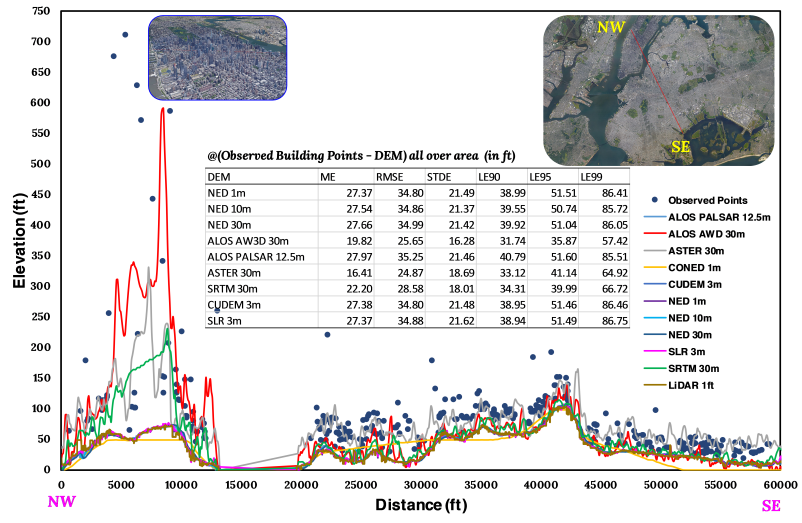

Figure 11. Topographic profile of all topography models with observed building elevations, Satellite Imagery

CLandsat/Copernicus, powered by Google Earth Pro

lowed bimodal distributions. All DEMs showed a similar mean error minimum value for ALOS AW3D, while ASTER showed a negative error. The RMSE and linear error were calculated for all considered DEMs with building elevations as a base dataset. The error matrices table is shown in Figure 11. The ALOS AW3D showed an overall minimum error with RMSE 25.65ft and LE90 31.74ft. All DEMs showed nearer error metrics, so there is not much difference observed between high-resolution DEMs (SLR 3m, CUDEM 3m, NED 1m) and medium resolution DEMs (ALOS AW3D 30m, ASTER 30m, SRTM 30m).

The profile was drawn from NW to SE, which crosses Manhattan midtown and Brooklyn county, where major urban sprawls are spread with high raised buildings. The profile section is shown in Figure 11. The figure shows that the ALOS AW3D shows the high raised building at 5000 to $1000 \mathrm{ft}$ distance. In between 5000 to $8000 \mathrm{ft}$, the observed building footprints showed high elevations, but in ALOS, ASTER, and SRTM were not identified. It is due to these data sets acquired during 1999-2000 (ASTER and SRTM), 2006 (ALOS AW3D), but these buildings were constructed after 2006. Other than ALOS AW3D, ASTER, and SRTM, the remaining DEMs have shown smooth surface profile with minor undulations. In between $5000 \mathrm{ft}$ to $8000 \mathrm{ft}$ SRTM given smooth profile while same spot ASTER and ALOS AW3D showed building undulations due to layover or shadow or foreshortening of radar signal.

\section{SUMMARY AND CONCLUSION}

This study is conducted to identify the suitable alternative freely available or medium resolution topography model for urban flood studies. To achieve the goal, different objectives include descriptive statistics of elevations and slopes for all DEMs; stream and watershed identification; contour generation; Topography Wetness Index analysis; and elevation discrepancies were performed over New York City. From the analysis of all objectives, it is identified that medium resolution DEMs are performed similarly to high-resolution DEMs in the context of urban extreme and nuisance floods. The ALOS AW3D shows an approximate correlation with the observed one, which may be due to the original product was acquired at $5 \mathrm{~m}$ spatial resolution. So it can observe in between buildings and other such artifacts, which can influence while averaging by resampling to produce $30 \mathrm{~m}$ data (Grohmann, 2018). Many studies explain that the data will not be lost after resampling the 
data from high resolution to medium resolution. The ALOS AW3D is a photogrammetry-based optical sensor dataset. In many places, data contain voids because optical signals cannot penetrate through clouds and have scene mismatch issues. In open places, ALOS AW3D, ASTER, and SRTM show similarly, which is due to the response of land cover classes to radar or optical sensors is comparable (Grohmann, 2018). The ASTER has also shown good agreement with LiDAR and observed elevations at the selected study area. However, in many places, it is reported that ASTER contains noise introduced during the dataset processing because artifacts are inherent to the automatic processing of optical imagery. The SRTM also performed well, but it is observed that it has given voids around the artifacts. The radar signal shadow, layover, and foreshortening might have caused the voids in the dataset. SRTM also created the stripping error due to uncompensated oscillations in its mast (Farr et al., 2007, Simard et al., 2016). The ALOS PALSAR performed very poorly even though having $12.5 \mathrm{~m}$ spatial resolution. Finally, it can be concluded that the ALOS AW3D can be used for urban flood modeling in the place of high-resolution DEMs. It is available in most of the cities freely, and the base data is acquired at $5 \mathrm{~m}$ spatial resolution, so it can store more of the urban features as it is in $5 \mathrm{~m}$ resolution.

\section{REFERENCES}

Chen, Y., Zhou, Q., 2013. A scale-adaptive DEM for multi-scale terrain analysis. International Journal of Geographical Information Science, 27(7), 1329-1348. doi.org/10.1080/13658816.2012.739690.

Chow, T. E., Hodgson, M. E., 2009. Effects of lidar postspacing and DEM resolution to mean slope estimation. International Journal of Geographical Information Science, 23(10), 1277-1295. doi.org/10.1080/13658810802344127.

Farr, T. G., Rosen, P. A., Caro, E., Crippen, R., Duren, R., Hensley, S., Kobrick, M., Paller, M., Rodriguez, E., Roth, L., Seal, D., Shaffer, S., Shimada, J., Umland, J., Werner, M., Oskin, M., Burbank, D., Alsdorf, D., 2007. The Shuttle Radar Topography Mission. Reviews of Geophysics, 45(2). doi/abs/10.1029/2005RG000183.

Fereshtehpour, M., Karamouz, M., 2018. DEM Resolution Effects on Coastal Flood Vulnerability Assessment: Deterministic and Probabilistic Approach. Water Resources Research, 54(7), 4965-4982. doi.org/10.1029/2017WR022318.

GRASS Development Team, 2019. Geographic Resources Analysis Support System (GRASS GIS) Software. Open Source Geospatial Foundation, USA.

Grohmann, C. H., 2015. Effects of spatial resolution on slope and aspect derivation for regional-scale analysis. Computers \& Geosciences, 77, 111 - 117. doi.org/10.1016/j.cageo.2015.02.003.

Grohmann, C. H., 2018. Evaluation of TanDEM-X DEMs on selected Brazilian sites: Comparison with SRTM, ASTER GDEM and ALOS AW3D30. Remote Sensing of Environment, 212, 121 - 133. doi.org/10.1016/j.rse.2018.04.043.

Kelleher, C., McPhillips, L., 2020. Exploring the application of topographic indices in urban areas as indicators of pluvial flooding locations. Hydrological Processes, 34(3), 780-794. doi.org/10.1002/hyp.13628.
Krieger, G., Moreira, A., Fiedler, H., Hajnsek, I., Werner, M., Younis, M., Zink, M., 2007. TanDEM-X: A Satellite Formation for High-Resolution SAR Interferometry. IEEE Transactions on Geoscience and Remote Sensing, 45(11), 3317-3341. 10.1109/TGRS.2007.900693.

Li, J., Wong, D. W., 2010. Effects of DEM sources on hydrologic applications. Computers, Environment and Urban Systems, 34(3), 251 - 261. doi.org/10.1016/j.compenvurbsys.2009.11.002.

Maidment, D. R., Morehouse, S., 2002. Arc Hydro: GIS for water resources. ESRI, Inc.

Pipaud, I., Loibl, D., Lehmkuhl, F., 2015. Evaluation of TanDEM-X elevation data for geomorphological mapping and interpretation in high mountain environments - A case study from SE Tibet, China. Geomorphology, 246, 232 - 254. doi.org/10.1016/j.geomorph.2015.06.025.

Qin, L., Xu, W., Tian, Y., Chen, B., Wang, S., 2018. A River Channel Extraction Method for Urban Environments Based on Terrain Transition Lines. Water Resources Research, 54(7), 4887-4900. doi/abs/10.1029/2018WR023095.

Sanders, B. F., 2007. Evaluation of on-line DEMs for flood inundation modeling. Advances in Water Resources, 30(8), 1831 - 1843. doi.org/10.1016/j.advwatres.2007.02.005.

Simard, M., Neumann, M., Buckley, S., 2016. Validation of the new srtm digital elevation model (nasadem) with icesat/glas over the united states. 2016 IEEE International Geoscience and Remote Sensing Symposium (IGARSS), 3227-3229.

Tachikawa, T., Kaku, M., Iwasaki, A., Gesch, D. B., Oimoen, M. J., Zhang, Z., Danielson, J. J., Krieger, T., Curtis, B., Haase, J. et al., 2011. Aster global digital elevation model version 2summary of validation results. Technical report, NASA.

Tadono, T., Nagai, H., Ishida, H., Oda, F., Naito, S., Minakawa, K., Iwamoto, H., 2016. GENERATION OF THE $30 \mathrm{M}$ MESH GLOBAL DIGITAL SURFACE MODEL BY ALOS PRISM. International Archives of the Photogrammetry, Remote Sensing \& Spatial Information Sciences, XLI-B4, 157-162. 10.5194/isprs-archives-XLI-B4-157-2016.

Zhang, K., Gann, D., Ross, M., Robertson, Q., Sarmiento, J., Santana, S., Rhome, J., Fritz, C., 2019. Accuracy assessment of ASTER, SRTM, ALOS, and TDX DEMs for Hispaniola and implications for mapping vulnerability to coastal flooding. Remote Sensing of Environment, 225, 290 - 306. doi.org/10.1016/j.rse.2019.02.028. 